\title{
Clinical Findings Versus Imaging Studies in the Diagnosis of Infantile Hypertrophic Pyloric Stenosis
}

\author{
Dr.Ahmed kadhum mohammed FJMC( RAD) \\ fellowship of jordanian medical council (radiology)AL-karama teaching hospital \\ Dr.Haider Ibraheem Khaleel \\ Pediatric surgery Central Child Teaching Hospital of Baghdad \\ Dr.SirarQahtan.Hameed \\ Central child teaching hospital of Baghdad
}

\begin{abstract}
Background: Infantile hypertrophic pyloric stenosis is the commonest surgical cause of vomiting in early infancy and can be diagnosed clinically or by imaging studies.

OBJECTIVES: To assess the accuracy of clinical examination as compared with ultrasound and upper gastrointestinal contrast imaging in the diagnosis of infantile hypertrophic pyloric stenosis.

PATIENTS AND METHODS: A prospective study of 30 patients referred to the pediatric surgical department in pediatric teaching hospital in Erbil with a possible diagnosis of IHPS in the period from June 2006 to January 2009.

RESULTS: The male to female ratio was 3.28:1 . The mean age at onset was 29.5 days. The mean age at presentation was 48 days. Projectile vomiting was present in all infants (100\%). Pyloric mass was palpable in 21 infants (70\%). Visible peristaltic waves were noted in 12 infants (40\%). 27 patients $(90 \%)$ had gastric aspirate of more than $10 \mathrm{cc}$. Ultrasound examination was confirmatory in 29 patients $(96.6 \%)$. Barium study was positive in 27 cases $(90 \%)$ and different signs were noted with various percentages. All patients underwent Ramstedt's operation through right upper transverse incision. The average hospital stay was 3.5 days. The long term results in all the 30 cases were excellent.

CONCLUSION: A palpable pyloric mass with suggestive history is a sufficient indication for proceeding to surgical treatment without confirming diagnostic imaging studies. However, when physical findings alone are inconclusive, an upper gastrointestinal series or an abdominal ultrasound examination should be done because of their high sensitivity in identifying the underlying problems.
\end{abstract}

DOI: $10.7176 / \mathrm{JHMN} / 76-04$

Publication date:June 30th 2020

\section{Introduction}

Infantile hypertrophic pyloric stenosis (IHPS) is the commonest surgical cause of vomiting in early infancy and it occurs in about $3 / 1000$ live births ${ }^{(1,2,3,4)}$.

The diagnosis can be made clinically by careful abdominal examination after a suggestive history of projectile vomiting in an infant aged between 2 and 8 weeks with associated weight $\operatorname{loss}^{(1,3,5,6)}$. Visible gastric peristalsis is a supportive finding, while palpation of an olive shaped mass in the rights upper quadrant of the abdomen is diagnostic ${ }^{(5,6,7,8,9,10)}$. The availability of ultrasonography and barium studies, however, has raised a question about the best method of diagnosis. Godbole et al found that a palpable pyloric mass was detected in $80 \%$ of cases ${ }^{(11)}$ while similarly Charles et al suggested that diagnosis by mean of clinical methods is possible in $89 \%$ of cases $^{(12)}$. Such patient should be operated on without undergoing imaging studies ${ }^{(1,2,3,4,5,7,8)}$. In the absence of a palpable mass, the diagnosis should be obtained by either ultrasonography or barium study ${ }^{(1,2,3,5)}$.

In 1934 it was shown that pyloric stenosis could be confirmed by barium meal examination, and in 1977 Teele and Smith first described the use of ultrasound in the diagnosis ${ }^{(13,14)}$. These techniques have been relied on increasingly to evaluate vomiting infants. Frieda Hulka et al noted that $61 \%$ of patients in earliest group of her study and $96 \%$ of patients in the latest group underwent an imaging study and in $86 \%$ it was ordered by the referring physician $^{(15)}$, while Charles et al reported a 34\% increase in the use of imaging techniques between 1980 and $1984^{(12)}$. The increased reliance on imaging studies to diagnose infantile hypertrophic pyloric stenosis (IHPS) has been ascribed to inexperienced examiners not palpating a pyloric tumor and subsequently proceeding with further evaluation $^{(6)}$.

\section{OBJECTIVES Patients and Methods Patients and Methods}

A Prospective study of thirty patients with infantile hypertrophic pyloric stenosis was conducted in pediatric surgery department in pediatric teaching hospital in Erbil from June 2006 to January 2009.

All patients in this study underwent clinical, laboratory, radiological and sonographic evaluations. 
Detailed history was taken from their mothers concerning the age at onset and at presentation, sex, sequence of baby in the family and the nature, time, onset and type of vomiting, status of appetite, bowel motion, positive family history and drug history. For the clinical diagnosis we depended on:

1. Presence of projectile vomiting at the typical age.

2. Palpable pyloric mass.

3. Visible gastric peristalsis.

4. Volumetric measurement of residual gastric aspirate of more than $10 \mathrm{ml}$.

All patients underwent general examination, weight measurement and assessment of hydration status with particular attention to the baby's level of consciousness, urine output, eyes, fontanelles, skin turgor, mucous membrane and tearing. In addition to careful abdominal examination, mainly looking for the presence of palpable pyloric mass and visible gastric peristalsis. If the pyloric mass couldn't be palpated at the beginning, the following steps were taken:

1. We ensure that the infant is relaxed because if the infant is crying the pyloric mass will be difficult to be palpate, so we allow the child to suck a pacifier.

2. We examine with the knees and hips flexed to relax abdominal wall musculature.

3. We decompress the stomach with nasogastric tube (NGT) because if the stomach is full, the distended antrum will obscure the pylorus.

4. If the infant proves difficult to relax, we give the infant to the mother to be breast fed provided the stomach is empty at the commencement of the feed; the relaxation afforded by the breast feeding may enable the tumor to be felt before the stomach is refills and to observe for visible gastric contraction after feeding.

5 . We re-examined the infant after an interval of time and during sleeping.

In our study volumetric measurements of nasogastric aspirate performed in patients after fasting of at least one hour. A size 8 French nasogastric feeding tube was placed in the child's stomach. An aspirated of more than $10 \mathrm{~mL}$ of milky fluid implicated gastric outlet obstruction. While an aspirated of less than $10 \mathrm{~mL}$ suggested a medical cause for the emesis.

The sonographic features of IHPS were evaluated in all patients and the following three pyloric parameters were measured and evaluated: (1) thickness of the pyloric muscle, (2) diameter, and (3) length. For the sonographic examination a linear scanner with a $7 \mathrm{MHZ}$ transducer was used. Barium study was conducted in all patients with the collaboration of radiological department in our hospital. We empty the stomach by a nasogastric tube before and at the end of the study to eliminate the risk of aspiration. Different signs of pyloric stenosis were found and recorded. All the data and information were collected from these patients and were written in special data collecting form. Findings of history (projectile vomiting), clinical examination (palpable pyloric mass, visible gastric peristalsis and residual gastric aspirate of more than $10 \mathrm{ml}$ ), and ultrasound and barium meal were compared.

Once the diagnosis of IHPS was confirmed, serum electrolytes levels checked preoperatively and after correction of fluid and electrolytes imbalances, all patients underwent surgical operation under general anesthesia.

\section{Results}

A total of thirty patients with IHPS were included in the study and in all cases this was confirmed at operation. The male to female ratio was 3.28:1.The mean age at the onset of vomiting was 29.5 days, with the youngest 7 days and the oldest 91 days. The mean age at presentation was 48 days with a range of 24 to 112 days.12infants (40\%) were first born baby.

The single most common presenting symptoms were bile free Projectile emesis which was present in all cases $(100 \%)$. three of them $(10 \%)$ was coffee ground vomitus. The average duration of symptoms at presentation was about 18 days, with shortest period 10 days and the longest 28 days.Constipation was present in 20 infants $(66.6 \%)$. The mean weight at presentation was $3 \mathrm{~kg}$ with a range of $2.200 \mathrm{~kg}$ to $4 \mathrm{~kg}$. The state of hydration on admission was recorded as mild, moderate or severe, on this basis, $12(40 \%)$ infants were described as mildly dehydrated, $15(50 \%)$ as moderately so and $3(10 \%)$ as severely dehydrated.Jaundice was present in 3 cases $(10 \%)$.A firm spherical mass was noted on palpation of abdomen in 21 infants $(70 \%)$.Visible peristaltic waves were noted in only 12 infants (40\%).27 patients (90\%) had volume measurement of residual gastric aspirate of more than $10 \mathrm{cc}$ after one hour of fasting. Three patients had an aspirate of less than $10 \mathrm{cc} .36$ abdominal US were performed (US repeated in 6 cases). US examination was confirmatory in 29 patients (96.6\%).Increased muscle thickness of more than $4 \mathrm{~mm}$ in $29(96.6 \%)$ patients with both longitudinal and transverse images.Increased pyloric channel length of more than $16 \mathrm{~mm}$ in $27(90 \%)$ patients with the longitudinal image.Increased muscle diameter of more than $14 \mathrm{~mm}$ in 25(83.3\%) patients with both longitudinal and transverse image.There were initial 6 negative scans in patients proved to have IHPS on second scan and at surgery.US imaging therefore had a sensitivity of 96.6\%.Barium study was performed in all patients and different signs were noted .Distended stomach and delayed gastric emptying in 27 cases (90\%). Elongated pyloric channel in 24 infants (80\%).String sign in 22 infants (73.3\%).Double track sign in 5 infants (16.6\%).Caterpillar sign in 5 infants (16.6\%). Shoulder sign in 4 infants (13.3\%).9 patients (30\%) had hyponatremia (120-130 mmol/l) .6 patients $(20 \%)$ had hypokalemia $(2.1-3 \mathrm{mmol} / \mathrm{l}) .5$ 
patients $(16.6 \%)$ had hpochloremia $(75-88 \mathrm{mmol} / \mathrm{l})$. The mean duration of preoperative rehydration was 24 hours.All patients underwent Ramstedt's operation through right upper transverse incision.Oral intake was started 12-24 hours after operation in 29 cases. There was one case of intraoperative iatrogenic duodenal perforation but the lesion was recognized at the time of operation and the duodenal perforation was closed and a size 8 F NGT was inserted and the feeding was begun after 48 hours.Eighteen $(60 \%)$ patients developed postoperative emesis which all resolved after one day of operation.The long term results in all the 30 cases were excellent.The average stay in the surgical ward of the hospital was 3.5 days. thesia using classical Ramstdt's procedure .

\section{Discussion}

Infantile hypertrophic pyloric stenosis is a common surgical condition encountered in neonate and early infancy. The clinical features of bile free progressive projectile vomiting, visible gastric peristaltic waves, and an olive shaped palpable abdominal mass in the right upper quadrant are frequently diagnostic ${ }^{(6,21,22,24)}$.In this study, the mean age at onset was 30 days; this result is nearly similar to a study by Mark P who reported the mean age at onset about 4.5 weeks $^{(24)}$. while the mean age at presentation in this study was 48 days and this is not similar to most recent studies like a study by Macdessi and Oates who reported the mean age at presentation to be 40 days (6) and another study by Godbole who found the mean age at presentation was 38 days ${ }^{(11)}$. The mean duration of symptoms in this study was 18 days which is longer in comparison to 7-8 days in Macdessi and Oates study ${ }^{(6)}$, this is probably related to delayed diagnosis and referral by primary health care. The typical clinical presentation in all $(100 \%)$ of our patients was non bilious projectile vomiting, this is similar to a study by Gruber et al in which the projectile vomiting occurred in all cases in their study ${ }^{(29)}$. Three patients had coffee ground appearance presumably from associated gastritis or esophagitis, these three patients had persistent vomiting for more than two weeks. Weight loss and dehydration was noticed in all patients especially when vomiting was continuous for more than a few days, this result is similar to an old study by Mark $\mathrm{P}$ who found that dehydration was present in all 50 patients in his study ${ }^{(24)}$, but in a more recent study by Macdessi and Oates the incidence of dehydration was only $50 \%{ }^{(6)}$. This reflects the delayed presentation and diagnosis in our study in comparison to other recent studies. The detection of pyloric mass depends on the experience and patience of the examiner. In our study it was palpable in $21(70 \%)$ patients and it is similar to a study done by Godbole et al. who found palpable pyloric mass in $72 \%$ of his cases ${ }^{(11)}$ and according to lumhagen and Noble, the pyloric muscle tumor can be palpated in about $80 \%$ of IHPS patients by experienced clinicians ${ }^{(25)}$. While in a study by Mark p et al pyloric tumor was palpable in $48 \%{ }^{(15)}$. The presence of palpable pyloric mass was highly specific and sensitive for IHPS, unlike other clinical signs which are sensitive but not specific.Positive Feeding test with visible gastric peristalsis was positive in 12 Patient (40\%), this is similar to a study done by Macdessi and Oates who reported it in $47 \%$ of his cases ${ }^{(6)}$.In our study volumetric measurements of nasogastric aspirate was positive in 27 patients(90\%) after one hour of fasting, the other three patients had false negative findings. Although most patients develop vomiting after feeding, the residual gastric aspirates were present. Finkelstein and colleagues have already shown that IHPS was present in $91.7 \%$ of patients with $10 \mathrm{~mL}$ or more nasogastric aspirate, and gastroesophageal reflux was present in $85.7 \%$ of patients with less than $10 \mathrm{~mL}$ nasogastric aspirate in a population of 38 infants $^{(22)}$. In Mandell et al study, a volumetric measurement of $5 \mathrm{~mL}$ was used as the criteria to differentiate IHPS from non obstructive causes of vomiting and the study was able to diagnose $91 \%$ of IHPS cases $^{(23)}$.In addition to the history and clinical examination, confirmation of the diagnosis has traditionally been provided by barium study of the upper gastrointestinal tract. Barium study was greatly helpful when the hypertrophied pylorus was not demonstrated on ultrasound and to assess for other causes of vomiting. Barium meal was performed in all cases and different radiological signs were found. It was positive in 27 Patients, although the commonest finding in 27 patients (90\%) was distended stomach with delayed gastric emptying but it is the least reliable indicator of IHPS and can be seen with pylorospasm, gastric hypotonia, sepsis and ileus ${ }^{(21)}$. Other signs were elongated pyloric canal, String sign, double or triple track sign, caterpillar sign and shoulder sign which were positive in $80 \%, 73.3 \%, 16.6 \%, 16.6 \%$ and $13.3 \%$ respectively, these signs are specific for IHPS. Freund et al. found the incidence of radiological error to be $4.5 \%-11 \%{ }^{(31)}$ while Shuman et al. report the incidence of radiological error to be about $5 \%{ }^{(32)}$.However, radiological examinations involve exposure to ionizing radiation and the ingestion of contrast medium. In 3 patients with negative barium study, one of them had a palpable olive while all 3 patients had a positive US.In our study ultrasonographic examination was performed in all patients, and it revealed typical increased muscle thickness in $29(96.6 \%)$ of our patients, increased pyloric channel length in $27(90 \%)$ patients and increased muscle diameter in $25(83.3 \%)$ patients and false negative in 1 case $(3.4 \%)$. All these findings were performed by a radiologist experienced with pediatric radiology. Blumhagen and Coombs ${ }^{(30)}$ were the first to point out that pyloric muscle thickness is the most important sonographic parameter in the diagnosis of HPS. Blumhagen and Noble ${ }^{(25)}$ found that the degree of canal elongation to be considerably greater in patients with IHPS. Of the three parameters diameter, thickness and length, muscular wall thickness must be considered to be the most precise in sonography ${ }^{(14)}$. In equivocal cases, however, diameter and length may be helpful in confirming the diagnosis ${ }^{(14)}$. In our study US examination was confirmatory in 29 patients $(96.6 \%)$ and this is similar to a Godbole et al study in which the diagnosis of HPS was confirmed by ultrasound in 
$97 \%{ }^{(11)}$ and similarly Gibbs et al found that ultrasound were diagnostic in $97 \%{ }^{(29)}$. Although it is a non invasive, easy to perform, with direct visualization of the hypertrophied muscle and no risk of radiation or aspiration but it is more operator dependent ${ }^{(14)}$ and in six patients the initial US were negative but they were positive on the second US performed by experienced ultrasonographer.A comparison between clinical findings and both ultrasound and barium meal were made. The clinical findings (projectile vomiting in $100 \%$, palpable mass in $70 \%$, visible gastric peristalsis in $40 \%$ and residual gastric aspirate of more than $5-10 \mathrm{ml}$ in $90 \%$ ) were less sensitive than the ultrasound $(96.6 \%)$ and barium study (90\%) and this depended on some factors:

1. Clinical examination should be performed by experienced surgeon or clinician and the infant should be calm with empty stomach.

2. Ultrasound although it is highly sensitive but it is more operator depended.

3. Barium meal of some patients are not specific e.g. only dilated stomach and delay emptying which can occurs in other conditions.

\section{Conclusion}

1. Clinical examination is the main step in the diagnosis of IHPS.

2. Palpation of the pyloric olive obviates the need for further diagnostic evaluation.

3. Palpation of olive mass is not always successful depending on factors such as experience of the examiner, the presence of gastric distension and a calm infant.

4. Volumetric measurement of residual gastric aspirate in neonates and infants with non bilious projectile vomiting could enable differentiation between patients with IHPS and those with GER.

5. If the physical examination is negative or equivocal, sonography by an experienced sonographer must be performed. If the ultrasound finding is negative, then the infant should undergo upper gastrointestinal barium study.

6. Ultrasound is highly sensitive and specific and it is the method of choice for diagnosis of IHPS without a palpable olive.

\section{Recommendations}

1. Palpable mass with suggestive history is a sufficient reason for proceeding to surgical treatment without confirming diagnostic imaging studies.

2. Volumetric measurement of residual gastric aspirate need to be more evaluated on a larger number of patients with IHPS in comparism to control group to be considered as a standard sign in the diagnosis.

3. Ultrasound is the standard imaging study in diagnosing IHPS but the availability of experienced ultrasonographer is essential to achieve the best results.

4. Barium meal should not be performed unless the clinical examination is equivocal with negative ultrasound because of risk of radiation and aspiration

\section{References}

1. Brian F.Gilchrist and Marc S.Lessin. Pyloric stenosis. In Keith W.Ashcraft, George Whitfield Holcomb, J.Patrick Murphy eds. Pediatric surgery. Philadelphia: Elsevier Saunders, 2005; 407- 410.

2. Spencer W.Beasley. Pyloric stenosis. In Spencer W.Beasley, John M. Hutson, Alex W.Auldist eds. Essential pediatric surgery. London: Arnold, 1996; 39-44.

3. Edward M.Barksdale. Pyloric stenosis. In Moritz M. Ziegler, Richard G. Azizkhan, Thomas R. Weber eds. Operative pediatric surgery. New York: McGraw-Hill, 2003; 583-588.

4. Takao Fujimoto. Hypertrophic pyloric stenosis. In P.Puri, M.Hollwarth eds. Springer surgery atlas series: pediatric surgery. Heidelberg: Springer, 2006; 171-180.

5. Thomas T. Sato and Keith T. Oldham. Infantile hypertrophic pyloric stenosis. In: Lazar J Greenfield, Michael W. Mulholland, Keith T. Oldham, Gerald B. et al. Essentials of surgery: scientific principles and practice. Baltimore: Lippincott William and Wilkins, 1997; 1293-1296.

6. Macdessi J, Oates RK. Clinical diagnosis of pyloric stenosis: A declining art. BMJ 1993; 306: 553-5.

7. Mark D.Stringer. Infantile hypertrophic pyloric stenosis: principles of pediatric surgery. IN R.C.G.Russell, Norman S.Williams, Christopher J.k.Bulstrode. Baley and love's short practice of surgery. London: Arnold, 2004; 1425- 1426.

8. Matthew L. Moront. Hypertrophic pyloric stenosis. In Robert M.Arensman, Daniel A. Bambini, P. Stephen Almond eds. Pediatric surgery. Georgetown: Landes Bioscience, 2000; 85-88.

9. D.Mervyn Griffiths. Infantile hypertrophic pyloric stenosis. In Peter J.Morris, William C. Wood eds. Oxford textbook of surgery. Oxford: oxford university press, 2000; 2039-2041

10. Michael Irish. Hypertrophic pyloric stenosis: surgical perspective: 2003, May. Available from URL: http: //www.emedicine.com /ped/topics/2965.html.

11. Godbole P, Sprigg A, Dickson JA, Lin PC.Ultrasound compared with clinical examination in infantile 
hypertrophic pyloric stenosis. Archives of Disease in Childhood 1996; 75: 335-7.

12. Charles W. Breaux, Keith E.Georgeson, Stuart A. Royal and Adrian J.Curnow.Changing patterns in the diagnosis of hypertrophic pyloric stenosis.Pediatric 1988; 18:213-217.

13. Marta Hernanz Schulman. Infantile Hypertrophic Pyloric Stenosis. Radiology 2003; 227:319-331.

14. R. GruBner, G. Pistor, B. Abon-Touk, and G. Alzen .Significance of ultrasound for the diagnosis of hypertrophic pyloric stenosis. Pediatric Surgery International 1986; 1: 130-134.

15. Frieda Hulka, Timothy J., John R., and MarvinW. Envolution in the recognition of infantile hypertrophic pyloric stenosis. Pediatrics1997; 100:1-9.

16. Chandrasen Kr. Sinha, A.N. Gangopadhyay, Sakti P. Sahoo,S. Chooramani Gopal, D.K. Gupta and B.B. Gupta.Congenital hypertrophic pyloric stenosis at birth. Indian journal of pediatric1996; 63:413-414.

17. Cooper WO, Griffin MR, Arbogast P. very early exposure to erythromycin and infantile hypertrophic pyloric stenosis. Archives of pediatric and adolescent Medicine2002; 156: 647-650.

18. Phillip C.Guzzetta, Kathryn D.Anderson, R.Peter Altman, Kurt D.Newman and Jay J.Schnitzer. Pyloric stenosis. In F.Charles Brunicardi, Dana K.Anderson, Timothy R.Billiar, David L.Dun, John G.Hunter, Raphael E.Pollock. Schwart'z principles of surgery. New York: McGraw-Hill`s 2004; 1728-1729.

19. Danhurt. Infantile hypertrophic pyloric stenosis. In: Charles W. Mitchell. Radiology review manual. Baltimore: Lippincott William and Wilkins, 1999; 692-693.

20. Grace Brooke Huffman. Evaluating infants with possible pyloric stenosis:1999,November . Available from URL: http://www.aafp.org/afp/991101/tips/24.html. (Web site of American academy of family physician).

21. Marija Frkovic, Marina Seronja, Zeljka Perhoc, Vinka Barbaric, Melite Molhar, Jurica Vukovic. Diagnostic imaging of hypertrophic pyloric stenosis. Radiology and oncology 2001; 35:11-16.

22. Finkelstein MS, Mandell GA, Tarbell KV. Hypertrophic pyloric stenosis: volumetric measurement of nasogastric aspirate to determine the imaging modality. Radiology 1990; 177: 759-61.

23. Mandell GA, Wolfson PJ, Adkins ES, Caro PA,Cassell I, Finkelstein MS, et al. Cost -effective imaging approach to the nonbilious vomiting infant. Pediatrics 1999; 103: 1198-1202.

24. Mark P. Owens and John R.Beljan. Congenital Hypertrophic Pyloric Stenosis. California Medical journal 1973 May; 118: 33-37.

25. Blumhagen JD, Noble HGS . Muscle thickness in hypertrophic pyloric stenosis: sonographic determination.AJR1983;140:221-223

26. David Martin and James Backstrom. Pediatric radiology. In Phillip L.Glick, Richard H.Pearl, Michael S.irish, Michael G. Caty eds. Pediatric surgery secrets. Philadelphia: Hanley and Belfus, 2001; 292-293.

27. Jack O. Haller, Thomas L. Solvis, Aparna Joshi. Pyloric stenosis. In Ute Heilmann eds. Pediatric radiology. Heidelberg: Springer, 2005; 134-136.

28. Bindi Naik-Mathuria, Oluyinka O. Olutoye. Foregut abnormalities. Surgical Clinics of North America 2006; 86: 261-284.

29. Gibbs MK, Van Herrden JA, Lynn HB. Congenital hypertrophic pyloric stenosis. Surgical experience. Mayo Clinic Procedures1975 Jun; 50:312-316.

30. Blumhagen JD, Coombs JB .Ultrasound in the diagnosis of hypertrophic pyloric stenosis. Journal of Clinical Ultrasound 1981; 9:289-292.

31. Shuman FI, Darling DB, Fisher JH. The radiographic diagnosis of congenital hypertrophic pyloric stenosis. Journal of Pediatrics 1967; 71:70-74.

32. Freund H, Berlatzky Y, Katzenleson R, Schiller.M. Diagnosis Of pyloric stenosis. Lancet II 1976; 22: 473 476.

\section{Conclusion}

1. Clinical examination is the main step in the diagnosis of IHPS.

2. Palpation of the pyloric olive obviates the need for further diagnostic evaluation.

3. Palpation of olive mass is not always successful depending on factors such as experience of the examiner, the presence of gastric distension and a calm infant.

4. Volumetric measurement of residual gastric aspirate in neonates and infants with non bilious projectile vomiting could enable differentiation between patients with IHPS and those with GER.

5. If the physical examination is negative or equivocal, sonography by an experienced sonographer must be performed. If the ultrasound finding is negative, then the infant should undergo upper gastrointestinal barium study.

6. Ultrasound is highly sensitive and specific and it is the method of choice for diagnosis of IHPS without a palpable olive.

\section{Recommendations}

1. Palpable mass with suggestive history is a sufficient reason for proceeding to surgical treatment without 
confirming diagnostic imaging studies.

2. Volumetric measurement of residual gastric aspirate need to be more evaluated on a larger number of patients with IHPS in comparism to control group to be considered as a standard sign in the diagnosis.

3. Ultrasound is the standard imaging study in diagnosing IHPS but the availability of experienced ultrasonographer is essential to achieve the best results.

4. Barium meal should not be performed unless the clinical examination is equivocal with negative ultrasound because of risk of radiation and aspiration. 